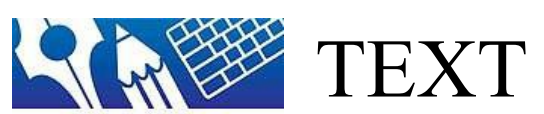

Australasian Association of Writing

Programs
Journal of writing and writing courses

ISSN: 1327-9556 | https://textjournal.scholasticahq.com/

TEXT Volume 25 No 1 April 2021

\title{
Editorial
}

\section{Welcome to the new TEXT website}

With this issue of TEXT we move from publishing the journal with our long-serving Australian host Netregistry, to publishing with Scholastica in the USA. Scholastica is specifically an open access academic journal host. That means TEXT will continue to be freely accessed by creative writers and scholars worldwide, and not locked up behind a paywall. Netregistry has provided us with excellent service for 15 years but they cannot, unfortunately, provide focused academic hosting services such as a refereeing system combined with free access.

Our new Scholastica-enabled TEXT web design optimises article pages for online discovery by embedding machine-readable article-level metadata. It features a responsive design that works on desktop and mobile, and the AAWP's new membership with Crossref has also allowed us to allocate a Digital Object Identifier (DOI) for TEXT articles as we publish them. A DOI is a string of numbers, letters and symbols used to permanently identify an article or document and will help our readers easily locate a document from a citation. Additional features at the new-look TEXT that we are looking forward to making fuller use of in the longer term include social media integrations, RSS feed option and new blog functionality.

The URL for TEXT will not change. It will stay as textjournal.com.au.

Moving a journal from one host to another is not a simple matter, especially when the process involves translating 25 years of HTML files into PDF files. TEXT was established in 1996 as one of the first online academic journals in the world, and just the second to be archived by the Australian National Library. Stemming from that, there is a lot of TEXT to be transferred to the new site. 
The solution the editors have settled upon in publishing with Scholastica is that more recent issues of TEXT will be available through the Scholastica site, and the rest of TEXT will be available via redirection to the old site. The process of editors converting historic TEXT for use on the new site (in PDF form) will go ahead as personpower and funds allow.

The TEXT editors thank the current AAWP Executive for their assistance with our digital renewal project.

We hope you enjoy exploring the new-look TEXT.

\section{- Nigel Krauth and Julienne van Loon}

\section{In this edition}

The April 2021 edition of TEXT features seven provocative scholarly contributions to the discipline, largely concerned with what could be considered an ethics of care. This septet is led by Julienne van Loon's (RMIT University) timely reflection on creative practice as 'nourishment', a notion van Loon draws from the work of political philosopher Corinne Pelluchon and applies to creative writing as a form of phenomenological 'radical sensing': a 'deeply attentive sensing' that not only nourishes the writer, but through the act of expression has the capacity to nourish others to sustain 'an ethics of life'. Originally presented as keynote to the Australasia Association of Writing Programs (AAWP) conference held at Griffith University, November 2020, van Loon offers her argument as reflection, as writing 'exercises', and with a 'polyvocal multi-authored "remix"' interlude as an illustration of her ideas.

The contribution from Cleo Mees takes a ficto-critical approach to the uncertainty, precarity and exploitation that characterises scholarly work for today's sessional creative writing academics. Poetic and poignant, Mees' article does not shy away from emotion: she examines the obligations writers feel (and have) to be happy or unhappy in their work and it is wonderful to see her frank search for meaning represented in TEXT. In a way, this article makes for an apt companion piece to the AAWP's Statement regarding Insecure Work in Higher Education.

Katherine Day's research sits in the allied area of publishing studies and brings an historically under-represented but valuable contribution to TEXT. Her intriguing and somewhat alarming research, drawn from her doctoral studies at the University of Melbourne, investigates the extent to which Australia's defamation laws have created a practice of implicit censorship of non-fiction writing in Australia. 
Continuing the theme of an ethics of care, in 'Haiku for recovery: An immersive workshop' Owen Bullock offers insights into his principles and practices in running haiku workshops at the University of Canberra as part of the Australian Defence Force Arts for Recovery, Resilience, Teamwork and Skills program. The article is intended as both a pedagogical method and demonstration, through Bullock's own reflections and the inclusion of participant haiku.

Jack Kirne's discussion of Olga Tokarczuk's novel Drive Your Plow Over the Bones of the Dead (2018) bolsters an argument for the multirealist mode as an agile method for writing about the climate crisis. Kirne's vision for multirealism emphasises its capacity to destabilise the ontological foundations of realism, and to make possible an imaginative space that can help us to radically reimagine the world and our engagement with it in the context of a rapidly changing planet.

Kári Gíslason finds limited capacity in the broader culture for critically valuing and genuinely supporting multi-modal authorship. His article seeks to address this gap by reflecting on the career of Clive James, whose contributions span poetry, memoir, writing for television, and criticism, among other modes. Gíslason asks: how might a more sympathetic approach to multimodal practice work to acknowledge the ways in which it can benefit writers, both artistically and practically?

Finally, 'Queerness, Form and Time: A dialogue through case studies from creative writing practice' is a remarkable collaboration of six scholars who engage in an experiment to explore the 'differences, contradictions, sympathies, antipathies, and strange resonances' between expressions of creative practice in relation to the 'notoriously slippery' yet personally significant notions of time and queerness, crucially, through experimentation with form. Contributors to this 'living discussion' include Ronnie Scott (RMIT University), Sholto Buck (RMIT University), J Butler (University of Melbourne), Jhoanna Lynn Cruz (University of the Philippines Mindanao), George Haddad (Western Sydney University) and Ann Lee (Independent scholar).

We hope you discover much creative and theoretical sustenance in this fascinating edition of TEXT.

— Ross Watkins and Julienne van Loon

\section{New Special Issue}


Published with this regular issue of TEXT is Special Issue Number 61, Writing Through Things: Writing the Past and Broken Things, edited by Debra Wain and Melanie Saward. Susan Pollock has noted, 'The object can hold an unexplored world, containing within it memory, emotion, and untapped creativity' (Pollock, 2011, p. 230). As the editors say in their Introduction, 'The scholars who present their work here in this Special Issue ... were invited to contribute their research in the form of a traditional article or creative work that explores the fission between the arena of the mind and the concrete, tangible things of the world around us; to explore that "unexplored world" of "memory, emotion, and untapped creativity".

\section{Work cited}

Pollock, S. (2011). The Rolling Pin. In S. Turkle (Ed.). Evocative Objects: Things We Think With. MIT Press, pp. 225-231. 